\title{
Non-Cooperative Target Identification Based on Singular Value Decomposition
}

\author{
Olga Hernán-Vega , Patricia López-Rodríguez , David Escot-Bocanegra , Raúl \\ Fernández-Recio and Ignacio Bravo
}

\begin{abstract}
Non-Cooperative Target Identification based on High Resolution Range Profiles is a key research domain in the Defense industry. In this paper a method based on the application of Singular Value Decomposition to a matrix of range profiles is defined. The decomposition is applied to reduce dimensionality and to accomplish recognition in the transformed domain. So as to confirm the feasibility of the methodology, identification experiments of profiles coming from electromagnetic simulations are conducted, revealing promising results.
\end{abstract}

Keywords: HRRP, NCTI, simulated/synthetic database, SVD.

\section{Introduction}

A High Resolution Range Profile (HRRP) is a one-dimensional radar image where the reflectivity of a target is projected onto the radar line of sight. Profiles are comprised of range bins and contain the distribution of the scattering centers of a target providing information about the target structure [1]. Non-Cooperative Target Identification/Recognition (NCTI/R) based on HRRP compares a profile, or collection of profiles, of a given target with the profiles included in a pre-loaded database. It has become a key research domain in the Defense industry since it provides the ability to recognize targets at long distances and under any weather condition [2-5].

The datasets employed in this document are obtained through numerical simulation of range profiles of a total of seven civil aircraft produced by an electromagnetic calculation software at defined trajectories. According, to that, to validate the approach considered here and since we intent to continue working in this line of research in the future using actual data from the very same measurement campaign, we first evaluate the methodology with simulated profiles. 
This work presents an approach to the NCTI problem based on the exploitation of Singular Value Decomposition (SVD) [6]. SVD has already been utilized in other studies for target recognition with HRRP as in [3], where SVD is used in order to create a database of range profiles templates, or in [7], where it is used for the noise reduction of simulated profiles. In this paper this technique is applied to matrices of consecutive HRRPs to reduce the classification difficulty. This is because SVD allows to work in a transformed domain where the main features are easier to extract hence reducing unwanted information such as noise. This way, the matrices of HRRPs are divided into subspaces and then the identification is accomplished by calculating the angle between subspaces.

\section{Methodology}

\subsection{Singular Value Decomposition}

SVD is a robust technique for the decomposition of any matrix into orthogonal basis spaces [6]. Let $X \in \Re^{N \times M}$ be a matrix of consecutive and aligned HRRPs of dimension $N \times M$ (assuming $N>M$ ), with $M$ being the total number of profiles and $N$ the number of range bins. This matrix $X$ can be decomposed into three orthogonal matrices $X=U S V^{T}$ where the columns of $U \in \Re^{N \times N}$ and $V \in \Re^{M \times M}$ are the $i$ th left and $i$ th right singular vectors of $X$ respectively. $S \in \Re^{N \times M}$ is a diagonal matrix containing the $p$ singular values of $X$ in descending order $\sigma_{1} \geq \sigma_{2} \geq \ldots \geq \sigma_{p} \geq 0$ with $p=\min \{N, M\}$.

The left singular vectors in $U$ span the orthogonal basis space in the range domain while the right singular vectors in $V$ span the basis space in the angle domain. Since HRRPs present the target reflectivity into the range domain, in this study only the left singular vectors $u_{i}$ will be used. Larger singular values, $\sigma_{i}$, imply larger contribution of the corresponding singular vector in forming the target signal. The Eckhart and Young theorem [6] guarantees that the top singular vectors with the highest singular values provide the best approximation of the data; the highest percentage of the energy is focused on the top ones. Therefore, the $N$-dimensional vector space can be divided into two subspaces, a dominant subspace, namely the signal subspace, and a subdominant subspace, namely the noise subspace. The singular vectors associated with the largest singular values are the basis that span the signal subspace while the rest will be discarded in the identification process since they are the basis that span the noise subspace. In this work $\eta$ is the energy threshold that divides the two subspaces and is defined by $\eta=\frac{\sum_{i=1}^{K} \sigma_{i}}{\sum_{i=1}^{p} \sigma_{i}}$.

In order to clarify the metrics used in this paper, Fig. 1 depicts the concept of subspace division and angle between subspaces. Let vectors $e_{1}, e_{2}$ and $e_{3}$ in Fig. 1 be the left singular vectors obtained after applying SVD to the test set (the set of HRRPs corresponding to the target to be identified). Imagine that according to their associated singular value, the $\eta \%$ of the energy is focused on $e_{1}$ and $e_{2}$, so they define the signal subspace while $e_{3}$ is discarded since it defines the noise subspace. In this example, $e_{1}$ and $e_{2}$ corresponds to the XY plane. In Fig. 1 the left singular vectors of the training set (the set of HRRPs of one of the targets in the database) are $u_{i}(i=1,2)$. According to that, the angle between them and their projection onto the 
signal subspace is defined to obtain the level of dependency. Taking into account the value of the angles, $u_{1}$ is closer to the XY plane than $u_{2}$, since the smaller the angle, the closer to the subspace $\beta<\alpha$.

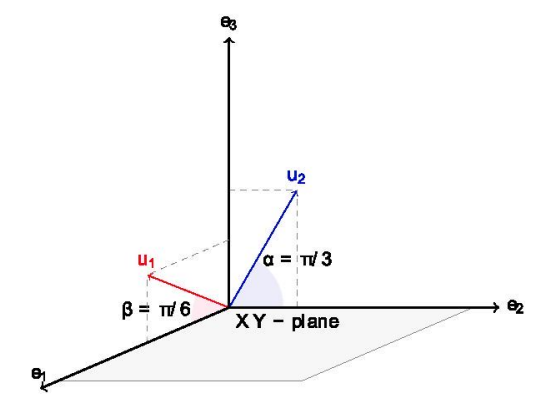

Figure 1: The signal subspace is defined by vectors $e_{1}$ and $e_{2}$

Denoting $X^{R}$ as the signal subspace containing the $K$ first left singular vectors of the test set and $u_{i}^{s}$ as the $i$ th left singular vector of the training set corresponding to target $s$; the accumulated angle between a singular vector in the training set $u_{i}^{s}$ and the signal subspace of the test set is given by (1).

$$
F 1_{s}(k)=\sum_{i=1}^{k} \operatorname{angle}\left(X^{R}, u_{i}^{s}\right) ; k=1, \ldots, K
$$

$F 1_{s}$ calculates the evolution of the angle formed by each singular vector and its projection onto the signal subspace resulting in a monotonically increasing function. The lowest final value of $F 1_{s}$ provides the recognized aircraft, $s$. In the case of $F 1_{s}$, the angle of every singular vector in the training set is considered equally important in the contribution to the final result. Let now vectors $u_{1}$ and $u_{2}$ in Fig. 1 be the left singular vectors obtained for two different aircraft $A$ and $B$ such that their associated singular values are:

$$
A=\left\{\begin{array}{l}
u_{1}^{A}=u_{1} \Longrightarrow \sigma_{1}^{A}=0.8 \\
u_{2}^{A}=u_{2} \Longrightarrow \sigma_{2}^{A}=0.2
\end{array} \quad B=\left\{\begin{array}{l}
u_{1}^{B}=u_{2} \Longrightarrow \sigma_{1}^{B}=0.8 \\
u_{2}^{B}=u_{1} \Longrightarrow \sigma_{2}^{B}=0.2
\end{array}\right.\right.
$$

where the superscript represents the aircraft $s$ to which the singular vectors and singular values are related. With the application of (1) there would be confusion in the result since: $F 1_{A}(K)=F 1_{B}(K)=\pi / 3+\pi / 6$. Due to the highest percentage of the energy is focused on the top singular values, not all singular vectors in the training set have the same importance. The angle between $u_{i}^{s}$ and $X^{R}$ should be weighted in a way that the singular value $\sigma_{i}^{s}$ associated with each $u_{i}^{s}$ sets the importance of this angle in the final solution. Obtaining low values of angle $\left(X^{R}, u_{i}^{s}\right)$ with the top singular vectors is more important than obtaining it with the ones that have a very low associated singular value. According to this, a new algorithm is given in (2). $F 2_{s}$ returns the accumulated weighted angle between the signal subspace of the test matrix and the singular vectors $u_{i}$ for each synthetic aircraft $s$ in the training set. 


$$
F 2_{s}(k)=\frac{1}{\sum_{j=1}^{p} \sigma_{j}^{s}} \sum_{i=1}^{k} \sigma_{i}^{s} \cdot \operatorname{angle}\left(X^{R}, u_{i}^{s}\right) ; k=1, \ldots, K
$$

In (2) $\sigma_{i, j}^{s}$ are the $p$ singular values obtained for each synthetic aircraft in the training set and, as in (1), angle $\left(X^{R}, u_{i}^{s}\right)$ is the angle between the test set signal subspace and each singular vector of the training set. Finally, the recognized aircraft, $s$, will be the one that minimizes the cost function (2). In the previous example, in contrast to $F 1_{s}$ the application of (2) would result in the identification of aircraft A since: $F 2_{A}(K)=1 \cdot\left(0.8 \cdot \frac{\pi}{6}+0.2 \cdot \frac{\pi}{3}\right)<F 2_{B}(K)=1 \cdot\left(0.8 \cdot \frac{\pi}{3}+0.2 \cdot \frac{\pi}{6}\right)$.

\subsection{Datasets}

In this research, two collections of computer models from 7 civil aircraft in different flightpaths are considered, a test set and a training set. These trajectories are taken from the ORFEO measurement campaign in which an S-band radar was employed [8]. Comparing to other studies, most of them tend to classify one profile a time, here NCTI process is carried out using a total number of 48 legs (sequence of consecutive HRRPs ordered in time). Each leg consists of 100 up to 300 consecutive, normalized and aligned profiles. The datasets of the seven aircraft are obtained for all the directions (aspect angles) contained in the legs by electromagnetic computation employing FASCRO, a Radar Cross Section (RCS) prediction code based on high frequency techniques (Physical Optics, PO, and Physical Theory of Diffraction, PTD) [9]. The profiles have been developed considering very aircraft as perfect electric conductors with no protruding elements. Taking into account the nature of the datasets, the main drawback of using simulated profiles instead of actual HRRPs is that the former implies an ideal identification scenario since the test and training samples have similar high Signal-to-Noise Ratio (SNR). In order to assess the feasibility of the approach in a actual possible scenario, a second case has been considered by adding noise before the creation of the test set, since actual HRRPs will suffer from noise and other undesired effects.

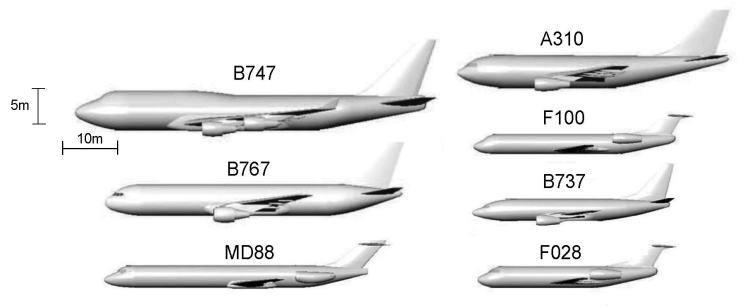

Figure 2: CAD models used for the RCS predictions: Boeing B747-400, Boeing B767-300, McDonnell Douglas MD88, Airbus A310-300, Fokker F100, Boeing B737-500 and Fokker F028

\section{Experimental Results}

Each simulated matrix of profiles for each aircraft and leg (test set) is compared with the rest of the aircraft for the same leg (training set) following the methodology and the two metrics 
presented in the previous section. The results are assessed and discussed here. As expected in the first case, if no noise is added the methodology gives a $100 \%$ of success rate so, in order to study the validity of the approach, the second case by adding noise has been considered. This intends to simulate the scenario of a more real identification system in which the data from the target to be identified will be more corrupted with noise. Several cases of SNR are analyzed with the purpose of demonstrating which proposed algorithm provides a higher feasibility. The addition of noise implies that the HRRPs in the test set are noticeably different from the profiles in the training set. An example of the differences can be found in Fig. 3 where the profiles of a Boeing 767 at a certain aspect angle with $-5 d B$ of SNR are depicted. As noticed, in Fig. 3 the dominant scattering center is reduced when a low SNR is added. This supposes a challenge for our approach so, for palliating it, normalization to unit energy of the profiles is applied to both sets (Norm-1 is employed). Then SVD is applied to a matrix of consecutive HRRPs and the rest of the methodology is developed as previously described.

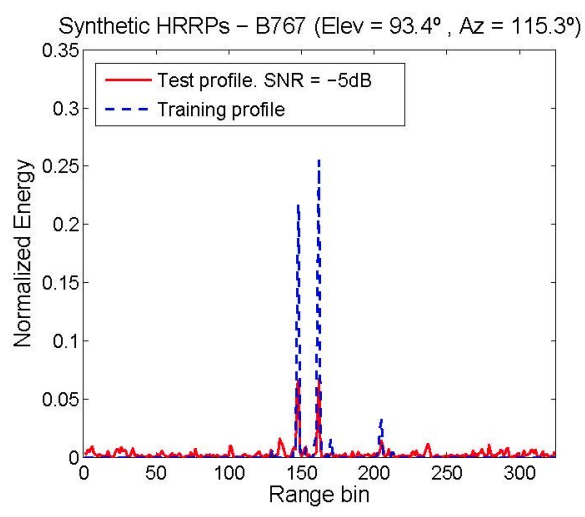

Figure 3: Difference between test and training sets

Identification rates of the 48 legs with a threshold of $\eta=0.9$ applying $F 1_{s}$ and $F 2_{s}$ can be seen in the Table 1. Taking into account the random nature of the noise, 20 experiments of each case are obtained; thus, the results given in Table 1 are the mean of these experiments.

Table 1: Recognition rates (\%) for the different cases of SNR

\begin{tabular}{ccccccccc}
\hline & $\mathbf{- 1 0 d B}$ & $\mathbf{- 5 d B}$ & $\mathbf{- 3 d B}$ & $\mathbf{0 d B}$ & $\mathbf{3 d B}$ & $\mathbf{5 d B}$ & $\mathbf{1 0 d B}$ & $\infty$ \\
\hline$F 1_{s}$ & 25.0 & 34.5 & 38.4 & 53.6 & 74.7 & 86.9 & 97.3 & 100 \\
$F 2_{s}$ & 89.3 & 91.9 & 93.1 & 93.8 & 94.6 & 95.5 & 98.2 & 100 \\
\hline
\end{tabular}

As expected in the results given in Table 1, a greater SNR means that more scatterers are visible and according to that, the lower the SNR, the worse the identification results obtained. This is a common behavior for both metrics. However, for this particular classification experiment, $F 2_{s}$ shows identification rates much better than those given by $F 1_{s}$ which has shown to be quite noise-dependent. Therefore, better recognition results are obtained when the singular values are used as weights in the cost function. This implies not only an improvement in the 
identification rate for the 7 simulated models of aircraft but also it demonstrates the robustness of the recognition system in a actual possible scheme.

\section{Conclusion}

In this paper a methodology and two metrics for HRRP target recognition based on SVD are shown. In order to study the feasibility of the approach, synthetic profiles are considered. As noted, the main drawback of using synthetic profiles with a very clean signature, is the ideal identification scheme since actual HRRPs will suffer from noise, clutter and other unwanted information. In order to check the feasibility of the identification system the addition of noise in the test set has been assessed. Regardless of the value of the SNR, it has been proven that finding the angle between singular vectors and signal subspaces is not sufficient for obtaining a good recognition performance. On the contrary, the addition of a weighting element (singular values) in the cost function produces a rise in the identification rate. Considering the dissimilarities between the test and training sets when noise is added to the former, the identification results obtained are also better if the singular values are considered. As demonstrated, the recognition performance has been improved by introducing weighting and this has also automatically increased the robustness of the system against noise. These results are quite promising and encouraging and future experiments with larger sets are expected to be conducted with the aim of finally using actual profiles as test sets.

\section{References}

[1] D. R. Wehner,“ High-Resolution Radar”, 2nd ed., Artech House, 1995.

[2] L. Chen and R. Chen, "A new radar target recognition method based on complex high resolution range profiles", in Microwave and Millimeter Wave Technology (ICMMT), 2012 International Conference on, 2012, vol. 2, pp. 1-4.

[3] V. Bhatnagar, A. Shaw, and R. Williams, "Improved automatic target recognition using singular value decomposition", in Acoustics, Speech and Signal Processing, 1998. Proceedings of the 1998 IEEE International Conference on, 1998, vol. 5, pp. 2717-2720.

[4] D. Zhou, X. Shen and Y. Liu, "Nonlinear subprofile space for radar HRRP recognition", Progress in Electromagnetics Research Letters, 2012, vol. 33, pp. 91-100.

[5] L. Du, H. Liu, Z. Bao, and J. Zhang, "Radar automatic target recognition using complex highresolution range profiles", Radar, Sonar Navigation, IET, 2007, vol. 1, no. 1, pp. 18-26.

[6] G. H. Golub and C. F. Van Loan, "Matrix computations", 3rd ed., Johns Hopkins University Press, 1996.

[7] K.-C. Lee, J.-S. Ou and M.-C. Fang, "Application of SVD noise-reduction technique to PCA based radar target recognition", Progress In Electromagnetics Research, 2008, vol. 81, pp. 447-459.

[8] R. van der Heiden and J. de Vries, "The ORFEO measurement campaign. TNO Report", 1996.

[9] J. Perez and M. Catedra, "Application of physical optics to the RCS computation of bodies modeled with NURBS surfaces", Antennnas and Propagation, IEEE Transactions on, 1994, vol. 42, no. 10 , pp. 1404-1411. 